\title{
Deep spectroscopy of the dwarf spheroidal NGC 185
}

\author{
Denise R. Gonçalves ${ }^{1,2}$, Laura Magrini ${ }^{3}$, Lucimara P. Martins ${ }^{4}$, \\ Ana M. Teodorescu ${ }^{5}$, Cintia Quireza ${ }^{1}$ and Gaia Lanfranchi ${ }^{3}$ \\ ${ }^{1}$ UFRJ - Observatório do Valongo, Ladeira Pedro Antonio 43, 20080-090 Rio de Janeiro, \\ Brazil. email: denise@astro.ufrj.br \\ ${ }^{2}$ Department of Physics and Astronomy, University College London, Gower Street, WC1E \\ 6BT London, UK \\ ${ }^{3}$ INAF - Osservatorio Astrofisico di Arcetri, Largo E. Fermi 5, I-50125 Firenze, Italy. \\ ${ }^{4}$ NAT - Universidade Cruzeiro do Sul, Rua Galvão Bueno 868, 01506-000 São Paulo, Brazil. \\ ${ }^{5}$ Institute for Astronomy, University of Hawaii, 2680 Woodlawn Drive, HI 96822 Honolulu, \\ USA.
}

\begin{abstract}
Dwarf galaxies are crucial to understand the formation and evolution of galaxies, since they constitute the most abundant galaxy population. Abundance ratios and their variations due to star formation and inflow/outflow of gas are key constraints to chemical evolution models. The determination of these abundances in the dwarf galaxies of the Local Universe is thus of extreme importance. NGC 185 is one of the four brightest dwarf companions of M31, but unlike the other three it has an important content of gas and dust. Interestingly enough, in an optical survey of bright nearby galaxies NGC 185 was classified as a Seyfert galaxy based on its integrated emission-line ratios in the nuclear regions. However, although its emission lines formally place it in the category of Seyfert it is probable that this galaxy does not contain a genuine active nucleus. In this contribution, we resume, firstly, our results of an empirical study of the galaxy, on which we characterise its emission-line population and obtain planetary nebulae abundance ratios (Gonçalves et al. 2012). And, secondly, we discuss our attempt to identify the possible ionization mechanisms for NGC 185 enlighting the controversial classification of this galaxy dwarf spheroidal (dSph) as well as Seyfert, via stellar population synthesis and chemical evolution modelling (Martins et al. 2011).
\end{abstract}

Keywords. Galaxies: dwarf, galaxies: abundances, Local Group, planetary nebulae: general, supernova: general, binaries: symbiotic

\section{Gemini imaging and spectroscopy}

We obtained deep $\mathrm{H} \alpha$ imaging followed by spectroscopic observations of the $\mathrm{H} \alpha$ emitting population in NGC 185 using GMOS-N at Gemini (Fig. 1a). In addition to the 5 bright planetary nebulae (PNe) previously found in the galaxy (Richer \& McCall 2008, and ref. therein) we found other, much fainter, PNe (see Gonçalves et al. 2011). We then re-calculated the electron temperatures and chemical abundances of the brightest ones, and derived, for the first time, their electron densities. The PN population properties are interpreted in terms of the chemical evolution of NGC 185, which suggests that it has suffered a significant chemical enrichment within the last 8 Gyr. We also discovered the first symbiotic star in the galaxy (Fig. 1c) and enlightened the properties of a supernova remnant located close to the centre of NGC 185 (Fig. 1b). 

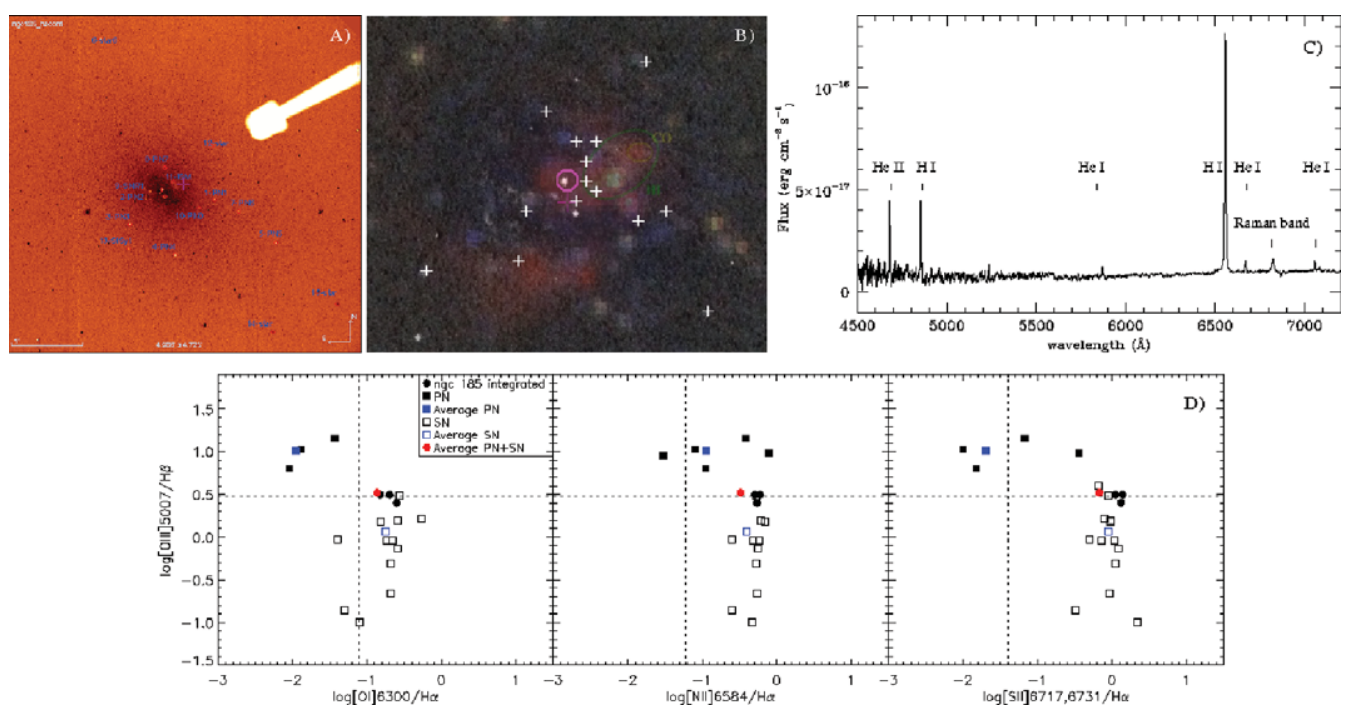

Figure 1. A) shows the position of our sample in the $5.5 \times 5.5 \operatorname{arcmin}^{2}$ continuum subtracted $\mathrm{H} \alpha$ image at the center of NGC 185. B) Shows the $\left(1.3 \times 1.3 \mathrm{arcmin}^{2}\right) \mathrm{H}$-continuum image with NGC 185-SNR-1, the circle, superposed on the IRAC three-colour image that highlights the fifteen bright blue young stars ('+' marks) with ages of $\sim 100 \mathrm{Myr}$, whose formation was probably induced by this SNR. C) The optical GMOS spectrum of the symbiotic star, NGC 185-SySt-1, which shows the $\mathrm{H}$ and He recombination lines, plus the Raman scattering at $6830 \AA$, only know to exist in symbiotic stars. D) The diagnostic diagram for NGC 185, based on the comparison of high- and low-excitation lines. Dotted lines give the division between active and non-active galaxies. Seyfert galaxies should be in the top right corn of all these plots.

\section{Faking a Seyfert galaxy}

NGC 185 was not detected in radio surveys either in $6 \mathrm{~cm}$ or $20 \mathrm{~cm}$, or X-ray observations, which might mean that the Seyfert-like line ratios may be produced by stellar processes. We discussed the possibility of the line emissions being produced by $\mathrm{PNe}$, using the deep spectroscopy observations discussed above. Although the fluxes of the PNe are high enough to explain the integrated spectrum that lead to its classification as a active galactic nuclei (Ho et al. 1997), their line ratios are far from the values for the Seyfert galaxy. We proposed a mixture of supernova remnants and PNe as the source of the ionization, and we show that a composition of these two objects do mimic Seyfertlike line ratios (Fig. 1 D). We used chemical evolution models (Lanfranchi \& Matteucci 2003), constrained by the abundance ratios above, to predict the supernova rates and to support the idea that these supernova remnants should be present in the galaxy (Martins et al. 2011).

\section{References}

Gonçalves D. R., Magrini L., Martins L. P., Teodorescu A. M., \& Quireza C. 2012, MNRAS, 419,854

Ho L., Filippenko A. V., \& Sargent W. L. W. 1997, ApJS, 112, 315

Lanfranchi G. \& Matteucci F. 2003, MNRAS, 345, 71

Martins L. P., Lanfranchi G., Gonçalves D. R., Magrini L., Teodorescu A. M., \& Quireza C. 2011, MNRAS, submitted

Richer M. G. \& McCall M. L. 2008, ApJ, 684, 1190 